\title{
ORC binding, gene amplification, and the nature of metazoan replication origins
}

\author{
Allan C. Spradling ${ }^{1}$ \\ Howard Hughes Medical Institute, Carnegie Institution of Washington, Baltimore, Maryland 21210 USA
}

Genomic DNA replication is a critical process shaped by strong evolutionary pressures. Rapid replication can give a viral or cellular genome an immediate advantage over its competitors as long as requisitely high accuracy is not sacrificed. Prokaryotes employ an extremely fast, accurate DNA replication mechanism utilizing sophisticated multiprotein machines (Kornberg and Baker 1992; Baker and Bell 1998). Genome duplication is controlled by the assembly and activation of bidirectional replication forks at specific replication origins, under the control of initiator proteins that bind origins sequence specifically. For example, Escherichia coli oriC binds multiple copies of the initiator protein, dnaA, at repeated motifs. In an ATP-dependent manner dnaA then locally unwinds the DNA, facilitates helicase loading, and organizes the assembly of polymerases, primases, and other components of the replication fork. Special proteins are required to assemble and maintain this unwieldy machinery. Thus, loading the ring-shaped six-protein dnaB helicase around the DNA requires dnaC, whereas proteins such as tau probably act as scaffolds, organizing replication fork components (including polymerases).

Despite the greater complexity of eukaryotic chromosomes, work carried out in Saccharomyces cerevisiae indicates that its genome replicates via a strongly similar mechanism (Leatherwood 1998). Each yeast chromosome contains multiple discrete replication origins where prereplication complexes are assembled during late $\mathrm{M}$ and G1 phases. Fully formed, bidirectional replication forks are then activated during $S$ phase. Replication origins can be readily identified as autonomously replicating sequence (ARS) elements that support the propagation of extrachromosomal plasmids. Budding yeast origins are $\sim 100 \mathrm{bp}$ in size and share an A/T-rich ARS consensus sequence (ACS) that is essential for origin function. Six proteins that form the origin recognition complex (ORC) bind to the ACS in an ATP-dependent manner throughout the cell cycle (Bell and Stillman 1992). Beginning in late mitosis, Cdc6p interacts with origin-bound ORC to bring the six-protein MCM complex, a putative replicative helicase, onto the DNA (Aparicio et al. 1997; Perkins and Diffley 1997; Tanaka et

1'E-MAIL spradling@mail1.ciwemb.edu; FAX (410) 243-6311. al. 1997). In a late step requiring cyclin-dependent kinase (cdk) activity, Cdc45p is added to the prereplication complex, possibly as a scaffold component (Zou and Stillman 1999). Activation of another origin-associated kinase, Cdc $7 p$, by its regulatory subunit Dbf4p, probably serves as the final signal activating replication fork movement (Dowell et al. 1994; Hardy 1998; Brown and Kelly 1999). By the time prereplication complexes fire, high cdk activity precludes the assembly of new complexes. Because a checkpoint ensures that the cell will not divide until replication is complete, and high levels of cdk activity are not reversed until mitosis is nearly finished, the genome will be replicated once and only once per cell cycle.

\section{The metazoan enigma}

The demands on DNA replication in metazoan eukaryotes significantly exceed those of simpler organisms. The genomes of Drosophila or Xenopus are 15 or 250 fold larger, respectively, than $S$. cerevisiae and are rich in recombination-prone repetitive DNA. Yet they need to be faithfully copied during embryonic cleavage stages in only half the time required to duplicate the E. coli genome. Metazoan genomes must replicate as many as $10^{14}$ times to produce the cells for a large animal. Moreover, the replication process must be compatible with hundreds of different cell types that are undergoing diverse processes of patterning and differentiation. In particular, the intricate and specific constellations of chromatin proteins in these cells need to be preserved. Given the extraordinary demands metazoans place on their replication machinery, it would not be surprising if new mechanisms not seen in yeast and prokaryotes had evolved.

Whether metazoans actually do utilize novel mechanisms to control DNA replication has remained controversial for most of the last two decades. Homologs of virtually all the major protein components of the budding yeast cell cycle and replication machinery have been identified. For example, a six-protein ORC complex in Drosophila binds DNA and is required for DNA replication (Gossen et al. 1995; Landis et al. 1997; Chesnekov et al. 1999). All six MCM homologs have also 
been identified (Feger 1999). Studies of replication in Xenopus oocyte extracts reveal strong similarities between budding yeast and higher eukaryotes (Pasero and Gasser 1998). Indeed, it is hard to imagine that a complex set of interacting proteins would have evolved a new way to carry out the same basic process. Nonetheless, other evidence exists to suggest that replication in metazoans differs significantly from the budding yeast paradigm.

One of the fundamental differences between metazoan and budding yeast replication lies in the nature of the cis-acting elements required for origin initiation. Metazoan DNA synthesis initiates during $S$ phase at multiple sites, some of which are cell-type specific and parallel differences in gene expression. However, it has been difficult to identify and study specific replication origins and to link them to particular DNA sequences. In part this may reflect the fact that plasmid maintenance assays used to identify yeast ARSs, have not yielded positive results. Even in the 15-20 cases where origins have been mapped based on the chromosomal location of replicative intermediates (DePamphlis 1999) their nature remains ambiguous. Two-dimensional gel electrophoresis methods frequently reveal less specific initiation sites and suggest that replication begins within a broad 'initiation zone' rather than at one or a few specific sites. In budding yeast both methods give consistent results, and the explanation for these discrepancies remains elusive. Moreover, metazoan replication appears to be influenced by chromatin structure on an even larger scale. Multiple replication origins sometimes appear to be clustered within nuclei into large 'replication factories' (Newport and Yan 1996). It has even been suggested that at the sequence level there are no preferred initiation sites, and that origin usage is determined in an entirely epigenetic manner by higher order chromatin structure and interactions with the nuclear matrix (Gilbert 1998). Consistent with this view, extracts from Xenopus oocytes can replicate virtually any added DNA once and only once, suggesting specific cis-acting sequences are nonessential for metazoan replication.

\section{The question of ORC binding specificity}

One key approach to assessing the importance of specific DNA sequences in initiation is to analyze the DNA binding specificity of eukaryotic ORC proteins, whose role as initiator proteins is presumably conserved. The budding yeast paradigm predicts that the ORC complex should preferentially bind to specific DNA sequences found within functional replication origins, whereas in a chromatin-based model, ORC should bind widely distributed sequences. Previous studies have failed to resolve this issue. For example, reconstituted Drosophila ORC complexes were not observed to bind to specific sequences (Chesnikov et al. 1999). The Schizosaccharomyces pombe Orc4 protein does show affinity for the ars1 origin (Chaung and Kelly 1999), but the specificity of this binding remains unclear.

Consequently, the paper in this issue by Austin et al. is of particular interest. The authors show for the first time that Drosophila ORC binds sequence specifically to regulatory elements known as ACE3, ACE1, and AER-d that have been implicated genetically in controlling replication initiation during eggshell (chorion) gene amplification. Specific association between ORC and replicator sequences was observed both in vivo and, using purified $A C E 3$ and $A E R-d$ DNA in vitro. Moreover, binding in vitro depended on the presence of ATP, like ORC binding to yeast ARS elements. In vivo, ORC associated with sequences extending several $\mathrm{Kb}$ from ACE3, and formed a doughnut-shaped structure that was visualized by immunofluorescence microscopy. Finally, in the absence of $A C E 3$, both ORC binding and DNA amplification were abolished.

This work provides the strongest evidence to date that sequence-specific recognition by ORC plays a role in activating at least some metazoan replicons. However, one might consider whether the nature of the amplification control sequences recognized by Drosophila ORC are suitable as models for normal replication origins. During a specific period of Drosophila oogenesis beginning at stage 10B, ovarian follicle cells amplify two specific genomic regions containing clusters of chorion genes by supporting extra rounds of replication initiation and bidirectional fork elongation (for review, see Orr-Weaver 1991; Calvi and Spradling 1999). Amplification of the third chromosome gene cluster is strongly dependent on $A C E 3$, a small cis-regulatory element located about 1.5 $\mathrm{Kb}$ from $A E R-d$, another cis-regulator that probably serves as the major replication origin. A strong cis-regulatory sequence involved in amplification of the first chromosome cluster, ACE1, has been less well localized. Unlike normal replication, initiation events during amplifcation are not limited to once per cell cycle. Moreover, late-stage follicle cells are polytene and most amplification takes place after the rest of the genome has ceased replicating.

Despite these differences, recent studies have documented a close relationship between chorion gene amplification and cell cycle-regulated chromosomal replication. First, a detailed examination of the follicle cell cycles indicated that amplification occurs during a final $S$ phase that is under the control of Cyclin E (Calvi et al. 1998). Inactivation of Cyclin E by misexpressing the p27like gene dacappo in follicle cells causes an immediate block to amplification. Thus, amplification, like normal replication, appears to require cdk activity. Second, mutations have been identified in E2F and DP that selectively block amplification, indicating that these two $\mathrm{S}$ phase initiator proteins required for normal replication are also needed for amplification (Royzmann et al. 1999). Third, the fact that 16 sister chromatids lie in close proximity in the polytene follicle cells allows proteins located at the amplification sites to be visualized by immunofluorescence microscopy prior to and during amplification. ORC1 and ORC2 protein can both be seen to localize to the sites where amplification will take place during stage 10A, just prior to the onset of the bulk of amplification (Asano et al. 1999; Royzmann et al. 1999), but are absent in some E2F and DP mutants. 
Further evidence that amplification utilizes normal replication machinery comes from studies of additional mutations that block amplification. A class of at least ten genes have been identified in which female-sterile mutations disrupt chorion gene amplification, causing viable adult females to produce thin eggshells. Amplification mutations probably select for leaky alleles of genes that are specifically involved in replication initiation. A twofold reduction in the rate of initiation may be compensated for during normal replication by an increase in the length of $S$ phase, but probably causes an exponentially increasing deficit in gene copy number during the short, fixed time period allotted for amplification. In this sense, amplification mutants resemble mutants selected for defective plasmid or minichromosome maintenance (Hogan and Koshland 1992; for review, see Tye 1999). The first amplification gene isolated, k43, encodes ORC2 (Landis et al. 1997). This confirms that ORC2 protein participates in amplification in vivo. Recently, another gene, chiffon, was characterized and found to contain a segment that is related to $S$. cerevisiae Dbf4 and its S. pombe counterpart Dfpl (Landis and Tower 1999). Metazoan homologs related to the Cdc7 kinase had been described previously, and Chiffon is a strong candidate for its regulatory subunit. Clearly, several major trans-acting components of normal DNA replication initiation also participate in the repeated initiation events that produce local amplification.

The cis-regulatory sequences within amplification control elements also generally resemble the few eukaryotic origin sequences outside of budding yeast that have been analyzed genetically (Clyne and Kelly 1995; Dubey et al. 1996; Aladjem et al. 1998; Kim and Huberman 1998, 1999|. These elements are larger than budding yeast origins: $3-4 \mathrm{~kb}$ in the case of the chorion locus, $0.5-2 \mathrm{~kb}$ in the case of $S$. pombe origins, and 3-4 kb in the case of the mouse globin region. Moreover, in both the chorion and globin replicators, normal flanking regions appear to contain redundant elements that act to enhance the overall efficiency of replication. In addition, one or more small zones with a strong effect on replication are found within the core region. Thus, the 430-bp $A C E 3$ segment was essential for amplification in the context of a 7.7-kb genomic fragment (Orr-Weaver and Spradling 1986). Three separate regions of $\sim 50 \mathrm{bp}$ in $S$. pombe ars3001 reduced its activity at least 30 fold (Kim and Huberman 1998). Furthermore, evidence of internal redundancy was observed in both elements (Orr-Weaver et al. 1989; Kim and Huberman 1998). Finally, the activity of both types of elements is subject to position effects when tested at ectopic genomic sites (deCicco and Spradling 1984; Aladjem et al. 1998). Normally, the mouse replicator requires an upstream locus control region (LCR), but was LCR-independent at two tested sites in CV-1 tissue culture cells. Position effects on amplification can also be buffered by a chromatin boundary element, namely su(Hw) binding sites (Lu and Tower 1998). Several of these characteristics have also been reported for the small elements controlling replication of the small rDNA minichromosomes in Tetrahymena macronuclei (Blomberg et al. 1998; Resichman et al. 1999).

The sequences within ACE3 and other eukaryotic origins also exhibit some similarities. Origins in $S$. pombe are frequently $\mathrm{A} / \mathrm{T}$-rich, contain runs of $\mathrm{A}$ and $\mathrm{T}$ residues, and display internal repeats. The ACE3 region contains conserved sequences, including an 80-bp A/T-rich sequence that is $>90 \%$ identical between Drosophila melanogaster, D. grimshawi, and D. virlis (Swimmer et al. 1990). This is particularly striking given that these species are estimated to have diverged $>40$ million years ago and show no other comparable regions of homology throughout the rest of the locus. This region contains several of the characteristics cited above, as the first 21 bp form a near-perfect direct repeat, and there are runs of Ts throughout the sequence. However, it is not essential for amplification (Orr-Weaver et al. 1989). Most of this highly conserved segment (Fig. 1) is contained within a 104-bp DNA fragment that stimulated strong ORC binding to purified DNA (Austin et al. 1999), suggesting that it might be a direct target of ATP-dependent ORC binding. Sequence-specific ORC binding was also observed with another region, $A E R-d$, located $\sim 1.5 \mathrm{~kb}$ from $A C E 3$. In $D$. melanogaster, this region also contains an $A / T$ rich region but its sequence is not conserved in the other species. It is not known where replication initiates in the $D$. grimshawi and $D$. virilis clusters.

\section{Amplification: model or exception?}

These observations argue that the chorion gene cluster replicates under cell cycle control using at least some

\section{D. melanogaster \\ D. grimshawi \\ D. virilis}

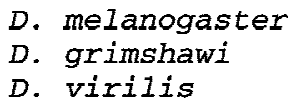

\begin{abstract}
TTTATAATTT TATTGTAATT TTATCTCAAT TTTTTTTGCT TTTGTATATA TTTATAATTT TATTGTAATT TTATCGTAAC TTTTTTTGCT TTTGTATATA TTTATAATTT TATTGTAATT TTATCGTAAC TTTTTTTGCT TTTGTATATA
\end{abstract}

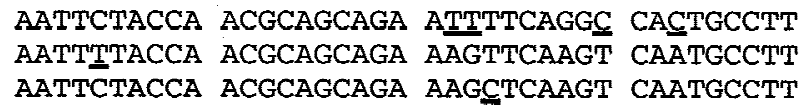

Figure 1. An alignment of DNA sequences within an $89 \mathrm{bp}$ conserved region located in a similar position several hundred base pairs upstream from the transcription start site of $s 18$ gene in the chorion gene clusters of three Drosophila species, D. melanogaster (X06257), D. grimshawi (X53422), and D. virilis (X53421). Nucleotides that differ from the consensus are underlined. 
homologs of budding yeast replication proteins. Moreover, the general characteristics of the cis-regulatory amplification control elements resemble the larger, more complex initiator sequences used by eukaryotes other than budding yeast. Despite this, it remains possible that the ORC binding observed by Austin et al. (1999) represents an exceptional situation. During stage 10A, ORC binding is observed only at the two major chorion loci that are about to amplify, as well as two minor loci, but not throughout the rest of the nucleus as would be expected during a normal cell cycle (Royzmann et al. 1999). ORC binding to the chorion loci just prior to amplification may require an exceptional, sequence-specific mechanism rather than the chromatin-based mechanism used during normal replication. The highly conserved sequence within ACE3 may have been preserved to help load ORC complexes under these circumstances, but not during normal replication. Even if sequence-specific ORC binding is a property of amplification control elements but not normal origins, further study will nonetheless assist in understanding how ORC helps assemble competent replication forks on metazoan chromosomes.

It seems at least as likely, however, that the control elements studied by Austin et al. (1999) are essentially normal metazoan replicator elements that are acted upon by as-yet undetermined mechanisms in late follicle cells to cause repeated rounds of initiation. In this view, the sequence-specific ORC binding observed by Austin and colleagues would be a general property of metazoan replication origins. The unusual requirement of $A C E 3$ for ORC binding throughout the region might indicate that in larger, more complex origins, sequences that mediate initial ORC binding can reside at a larger distance from the eventual site of initiation than is commonly seen in budding yeast origins (Bielinsky and Gerbi 1998). Perhaps, ACE3 mediates the initial binding and loading of many ORC molecules that spread over several kilobases of surrounding DNA. Eventually, replication forks would be assembled and initiate at other sites within the affected region, particularly $A E R-d$.

This model gains support from several additional observations reported by Austin et al. (1999). The strength of the immunofluoresence signals suggested that large amounts of ORC are present on the chorion loci, even in stage 10A. ORC complexes could be cross-linked in vivo to sequences that were located several kilobases distant from ACE3. Moreover, ORC protein showed a striking doughnut-like organization on amplifying chorion loci in stage $10 \mathrm{~B}$.

If origins used during normal $\mathrm{S}$ phase do bind ORC, then the work of Austin et al. (1999) establishes an assay that should allow many additional origins to be identified elsewhere in the genome. If ORC is loaded onto an extended region surrounding sequence-specific binding sites at other metazoan replication origins, this might somehow explain the observed discrepancies between different methods of origin mapping. Whatever the answers to these particular questions, the prospect of finally resolving the enigma of metazoan DNA replication has clearly been amplified.

\section{References}

Aladjem, M.I., L.W. Rodewald, J.L. Kolman, and G.M. Wahl. 1998. Genetic dissection of a mammalian replicator in the human $\beta$-globin locus. Science 281: 1005-1009.

Aparicio, O.M., D.M. Weinstein, and S.P. Bell. 1997. Components and dynamics of DNA replication complexes in S. cerevisiae: Redistribution of MCM proteins and Cdc45p during S phase. Cell 91: 59-69.

Asano, M. and R. Wharton. 1999. E2F mediates developmental and cell cycle regulation of ORC1 in Drosophila. EMBO J. 18: $2435-2448$.

Austin, R.J., T. Orr-Weaver, and S.P. Bell. 1999. Drosophila ORC specifically binds $A C E 3$, an origin of DNA replication control element. Genes \& Dev. 13 (this issue).

Baker, T.A. and S.P. Bell. 1998. Polymerases and the replisome: machines within machines. Cell 92: 295-305.

Bell, S.P. and B. Stillman. 1992. ATP-dependent recognition of eukaryotic origins of DNA replication by a multiprotein complex. Nature 357: 128-134.

Bielinsky, A.K. and S.A. Gerbi. 1998. Discrete start sites for DNA synthesis in the yeast ARS1 origin. Science 279: 9598.

Blomberg, P., C. Randolph, C.H. Yao, and M.C. Yao. 1997. Regulatory sequences for the amplification and replication of the ribosomal DNA minichromosome in Tetrahymena thermophila. Mol. Cell. Biol. 17: 7237-7247.

Brown, G.W., and T.J. Kelly. 1999. Cell cycle regulation of Dfp1, an activator of the Hsk1 protein kinase. Proc. Natl. Acad. Sci. 96: 8443-8448.

Calvi, B.R., M.A. Lilly, and A.C. Spradling. 1998. Cell cycle control of chorion gene amplification. Genes \& Dev. 12: 734-744

Calvi, B.R. and A.C. Spradling. 1999. Chorion gene amplification in Drosophila: A model for metazoan origins of DNA replication and S-phase control. Methods 18: 407-417.

Chesnokov, I., M. Gossen, D. Remus, and M. Botchan. 1999. Assembly of functionally active Drosophila origin recognition complex from recombinant proteins. Genes \& Dev. 13: 1289-1296.

Chuang, R.Y., and T.J. Kelly. 1999. The fission yeast homologue of Orc4p binds to replication origin DNA via multiple AThooks. Proc. Natl. Acad. Sci. 96: 2656-2661.

Clyne, R.K. and T.J. Kelly. 1995. Genetic analysis of an ARS element from the fission yeast Schizosaccharomyces pombe. EMBO J. 14: 6348-6357.

deCicco, D.V. and A.C. Spradling. 1984. Localization of a cisacting element responsible for the developmentally regulated amplification of Drosophila chorion genes. Cell 38: 4554.

DePampilis, M.L. 1999. Replication origins in metazoan chromosomes: Fact or Fiction? BioEssays 21: 5-16.

Dowell, S.J., P. Romanowski, and J.F. Diffley. 1994. Interaction of Dbf4, the Cdc7 protein kinase regulatory subunit, with yeast replication origins in vivo. Science 269: 1243-1248.

Dubey, D.D., S.M., I.T. Todorov, and J.A. Huberman. 1998. Large, complex modular structure of a fission yeast DNA replication origin. Curr. Biol. 6: 467-473.

Feger, G. 1999. Identification and complete cDNA sequence of the missing Drosophila MCMs: DmMCM3, DmMCM6, and DmMCM7. Gene 27: 149-155.

Gilbert, D.M. 1999. Replication origins in yeast versus metazoa: Separation of the haves and the have nots. Curr. Opin. Genet. Dev.8: 194-199.

Gossen, M., D.T. Pak, S.K. Hansen, J.K. Acharya, and M.R. Botchan. 1995. A Drosophila homolog of the yeast origin 
recognition complex. Science 270: 1674-1677.

Hardy, C.F. 1998. Characterization of an essential Orc2p-associated factor that plays a role in DNA replication. Mol. Cell. Biol. 16: 1832-1841.

Hogan, E. and D. Koshland. 1992. Addition of extra origins of replication to a minichromosome suppresses its mitotic loss in cdc6 and cdc14 mutants of Saccharomyces cerevisiae. Proc. Natl. Acad. Sci. 89: 3098-3102.

Kim, S.M. and J.A. Huberman. 1998. Multiple orientation-dependent, synergistically interacting, similar domains in the ribosomal DNA replication origin of the fission yeast, $S$. pombe. Mol. Cell. Biol. 18: 7294-7303.

. 1999. Influence of a replication enhancer on the hierarchy of origin efficiencies within a cluster of DNA replication origins. J. Mol. Biol. 288: 867-882.

Korenberg, A. and T.A. Baker. 1992. DNA replication. Freeman, New York, N.Y.

Landis, G. and J. Tower. 1999. The Drosophila chiffon gene is required for chorion gene amplification, and is related to the yeast Dbf4 regulator of DNA replication. Development 126 4281-4293.

Landis, G., R. Kelley, A.C. Spradling, and J. Tower. 1997. The $k 43$ gene, required for chorion gene amplification and diploid cell chromosome replication, encodes the Drosophila homolog of yeast origin recognition complex subunit 2. Proc. Nat1. Acad. Sci. 94: 3888-3892.

Leatherwood, J. 1998. Emerging mechanisms of eukaryotic DNA replication initiation. Curr. Opin. Cell Biol. 10: $742-$ 747.

Lu, L. and J. Tower. 1997. A transcriptional insulator element, the $\mathrm{su}(\mathrm{Hw})$ binding site, protects a chromosomal DNA replication origin from position effects. Mol. Cell. Biol. 17: 2202-2206

Newport, J. and H. Yan. 1996. Organization of DNA into foci during replication. Curr. Opin. Cell. Biol. 8: 365-368.

Orr-Weaver, T. 1991. Drosophila chorion gnes: cracking the eggshell's secrets. BioEssays 13: 97-105.

Orr-Weaver, T.L. and A.C. Spradling. 1986. Drosophila chorion gene amplification requires an upstream region regulating s18 transcription. Mol. Cell. Biol. 6: 4624-4633.

Orr-Weaver, T.L., C.G. Johnston, and A.C. Spradling. 1989. The role of $A C E 3$ in chorion gene amplification. EMBO J. 8: 4153-4162.

Pasero, P. and S.M. Gasser. 1998. New systems for replicating DNA in vitro. Curr. Opin. Cell Biol. 10: 304-310.

Perkins, G. and J.F. Diffley. 1997. Nucleotide-dependent prereplicative complex assembly by Cdc6p, a homolog of eukaryotic and prokaryotic clamp-loaders. Mol. Cell 2: 23-32.

Reischmann, K.P., Z. Zhang, and G.M. Kapler. 1999. Long range cooperative interactions regulate the initiation of replication in the Tetrahymena thermophila rDNA minichromosome. Nucleic Acids Res. 27: 3079-3089.

Royzman, I., R.J. Austin, G. Bosco, S.P. Bell, and T.L. OrrWeaver. 1999. ORC localization in Drosophila follicle cells and the effects of mutations in $\mathrm{dE} 2 \mathrm{~F}$ and $\mathrm{dDP}$. Genes \& Dev. 13: $827-840$.

Swimmer, C., M.G. Fenerjian, J.C. Martinez-Cruzado, and F.C. Kafatos. 1990. Evolution of the autosomal chorion cluster in Drosophila. III. Comparison of the $s 18$ gene in evolutionarily distant species and heterospecific control of chorion gene amplification. J. Mol. Biol. 215: 225-235.

Tanaka, T., D. Knapp, and K. Nasmyth. 1997. Loading of an Mcm protein onto DNA replication origins is regulated by Cdc6p and CDKs. Cell 90: 649-660.

Tye, B.K. 1999. Minichromosome maintenance as a genetic assay for defects in DNA replication. Methods 18: 329-334.
Zou, L. and B. Stillman. 1998. Formation of a preinitiation complex by S-phase cyclin CDK-depdndent loading of Cdc45p onto chromatin. Science 280: 593-598. 


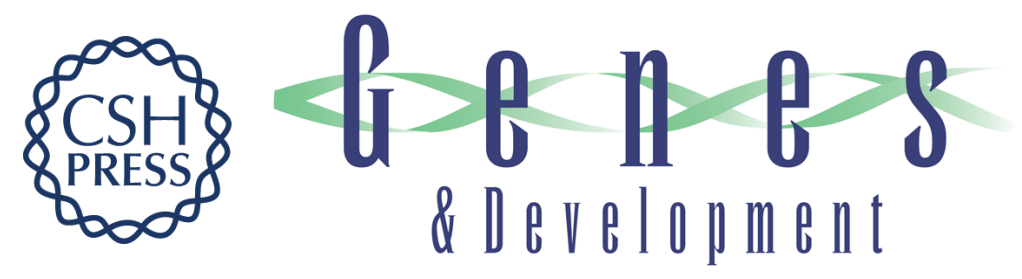

\title{
ORC binding, gene amplification, and the nature of metazoan replication origins
}

\author{
Allan C. Spradling \\ Genes Dev. 1999, 13:
}
References This article cites 36 articles, 17 of which can be accessed free at: http://genesdev.cshlp.org/content/13/20/2619.full.html\#ref-list-1

\section{License}
Email Alerting
Service
Receive free email alerts when new articles cite this article - sign up in the box at the top right corner of the article or click here.

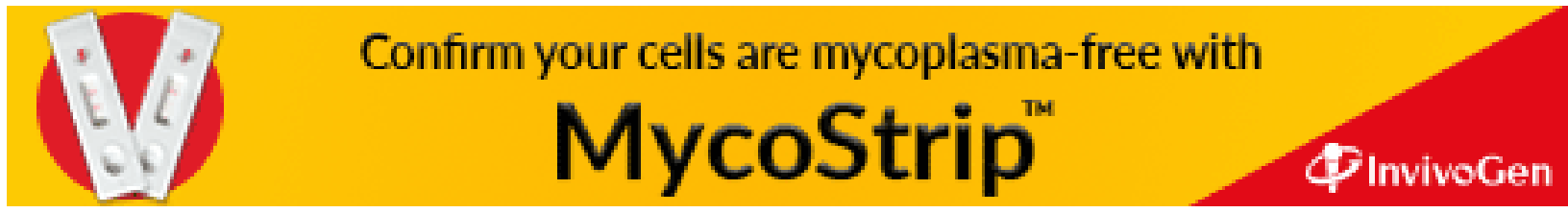

